\title{
Direct and Inverse Kinematic Problems of Tripod-Type Parallel Manipulator
}

\author{
Baurzhan K. NURAKHMETOV*, Kuanyshbek Z. SARTAYEV**, \\ Zhanat M. MYRZAGELDIYEVA* \\ *Almaty Technological University, Almaty, Kazakhstan \\ **Ekibastuz technical and engineering institute named after the academician K.Satpayev, Ekibastuz, Kazakhstan \\ Corresponding author: sshlyakhova55@yahoo.com
}

crossref http://dx.doi.org/10.5755/j01.mech.25.5.19523

\section{Introduction}

Unmanned aerial vehicle (UAV) platforms are a potential option for collecting near-earth data in real time [1-3]. UAV technology has matured from many years of using UAVs as hobby aircraft [4-5]. The main disadvantage of using drones in aerial photography is their small size and low weight. These characteristics, however, make the drones attractive in terms of cheap production. Besides, flexibility in application results in problems with the image quality, since the light device is extremely susceptible to vibrations. Even a stabilized camera captures and transmits an image that is not completely "clean". As a solution to this problem, we propose to use a gimbal based on a tripod-type parallel manipulator. Generally, this article is devoted to the process of building a theory and mathematical model of such a manipulator optimized for creating a gimbal for a UAV camera.

Briefly, the problem of optimizing parallel manipulator for the above-mentioned problem can be formulated as follows: a) this manipulator must be fast in order to react to high-frequency vibration, and b) the solution of direct and inverse kinematic problems should be optimized to solve these problems in real time, possibly with the least computing power. There is an additional consequence for the point b), since we have narrowed the scope of the problem to UAV camera stabilization. Solution mathematics can be simplified due to the predictable nature of high-frequency vibrations arising in a small size aircraft. In most cases, these will be harmonic oscillations caused by cyclic rotation of an air drive (propeller) and its engine (ICE or electric motor), as well as their harmonics. It is obvious that harmonic oscillations are predictable, and the flying vehicle does not often experience stochastic collisions with other solid bodies. Thus, we can use the harmonic nature of vibrations to simplify the solution of direct and inverse kinematic problems.

The kinematics of parallel manipulators is quite an interesting area in the field of robotic engineering [7], since, in contrast to traditional manipulators with chain structure, mechanisms with parallel kinematics have a number of advantages over the traditional mechanisms. Due to closed rigid structure of such manipulators, their bearing force increases, positioning accuracy increases, and fast operation speed increases by reducing the weight of mobile links. Thus, the structure of the parallel manipulators has good dynamic properties [8]. Gwinnett [9] had patented the mechanism with parallel kinematics, which was used as a stage for theatre. Later on, Gough [10] had proposed and implemented the idea of creating a mechanism with a parallel structure to increase the rigidity of the system. Stewart designed the platform of such a structure [11] intended to simulate flight trainer. At the present day Gough platform is the most common structure for flight simulators. Various construction algorithms of parallel mechanisms models with closed and open kinematic chains are considered in analyzed works. In order to determine the fixed position of the movable platform at the generalized coordinates in the direct kinematic problem and at the unknown generalized coordinates in the inverse kinematic problem, multi-sweep kinematic procedure of multistage core parallel manipulator with different kinematic pairs was applied.

\section{Direct kinematic problem}

During the solving of the direct kinematic problem for the manipulator mechanisms of parallel kinematics, for a specified kinematic scheme, geometric dimensions, and generalized coordinates, it is necessary to determine the position of the upper mobile platform. The kinematic chains of the parallel manipulator have fixed and time-varying parameters. In this tripod-type parallel manipulator (Fig. 1), in the capacity of time-varying parameters the progressive motion of cylindrical and rectilinear kinematic pairs is used.

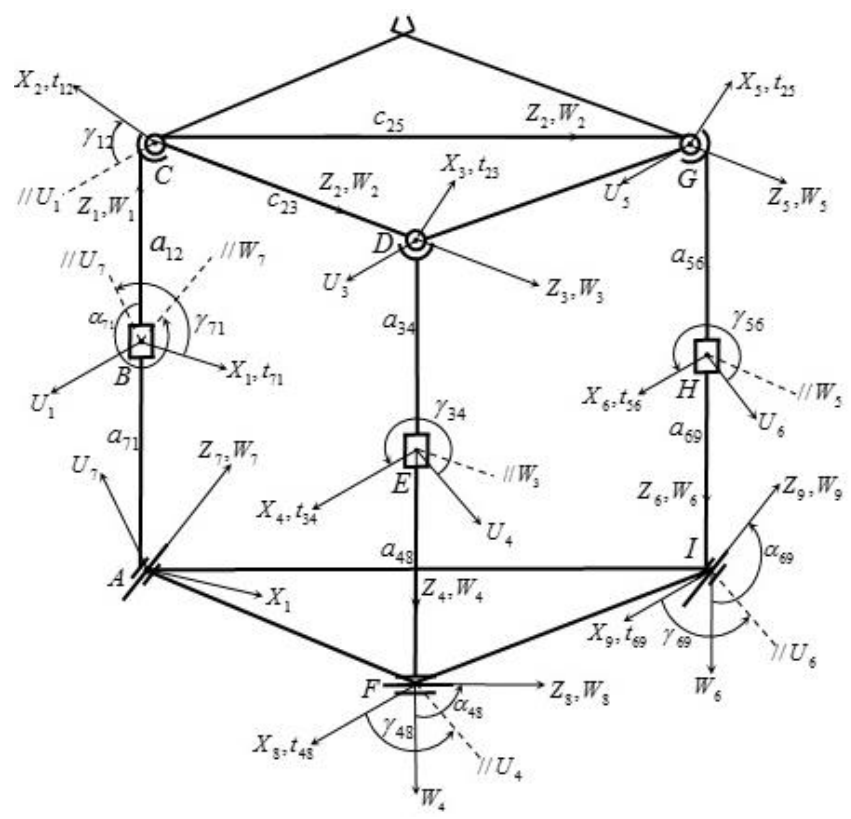

Fig. 1 Fixed and time-varying parameters of the tripod-type parallel manipulator 
To determine the fixed and time-varying parameters of parallel manipulator, the efficient machinespecific iterative method is used, which allows to accomplish an exhaustively analysis of the kinematics of any link, in any point of the parallel manipulator toward an inertial coordinate system. In this regard, each link of each kinematic pair of parallel manipulator is rigidly bounded; consequently, right rectangular coordinate system UVW and $\mathrm{XYZ}$. W and $\mathrm{Z}$ directions are converged and they are directed along the axis of turning shaft or prismatic motion of the kinematic pair. Directions of the other two pairs of axes $\mathrm{U}, \mathrm{V}$ and $\mathrm{X}, \mathrm{Y}$ may be directed at randomly, comply with the right Cartesian coordinate system UVW and XYZ.
Fig. 1 shows fixed and time-varying parameters of the tripod-type parallel manipulator. Present parallel manipulator has $n=9$ kinematic pairs, $n_{1}=7$ links, $m=6$ generalized coordinates, and $L=29$ circuits. For link $a$ of the circuit $l$, a pair of indices $(l, a)$ is assigned, and any link may be included in more than one circuit. Coordinate system rigidly bounded with each link in each kinematic pair, also has a pair of indices $(l, a)$.

Let us write the symbolic formula of $A B C D E F A$ and $A B C G H I A$ circuits' closure of the present tripod-type parallel manipulator: circuit 1:

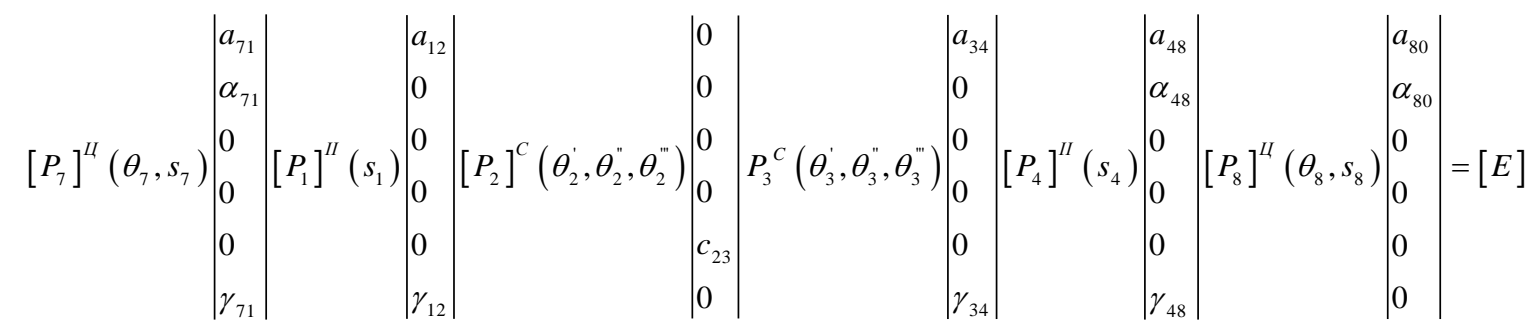

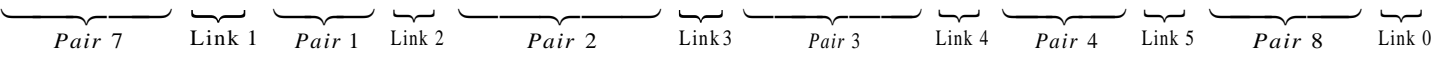

$$
\begin{aligned}
& {\left[P_{7}\right]^{L}\left(\theta_{7}, s_{7}\right)\left|\begin{array}{l}
a_{71} \\
\alpha_{71} \\
0 \\
0 \\
0 \\
\gamma_{71}
\end{array}\right|\left[P_{1}\right]^{I I}\left(s_{1}\right)\left|\begin{array}{l}
a_{12} \\
0 \\
0 \\
0 \\
0 \\
\gamma_{12}
\end{array}\right|\left[P_{2}\right]^{C}\left(\theta_{2}^{\prime}, \theta_{2}^{\prime \prime}, \theta_{2}^{\prime \prime \prime}\right)\left|\begin{array}{l}
0 \\
0 \\
0 \\
0 \\
c_{25} \\
0
\end{array}\right|\left[P_{5}\right]^{C}\left(\theta_{5}^{\prime}, \theta_{5}^{\prime \prime}, \theta_{5}^{\prime \prime \prime}\right)\left|\begin{array}{l}
a_{56} \\
0 \\
0 \\
0 \\
0 \\
\gamma_{56}
\end{array}\right|\left[P_{6}\right]^{I I}\left(s_{6}\right)\left|\begin{array}{l}
a_{69} \\
\alpha_{69} \\
0 \\
0 \\
0 \\
\gamma_{69}
\end{array}\right|\left[P_{9}\right]^{L I}\left(\theta_{9}, s_{9}\right)\left|\begin{array}{l}
a_{90} \\
\alpha_{90} \\
0 \\
0 \\
0 \\
0
\end{array}\right|=[E]}
\end{aligned}
$$

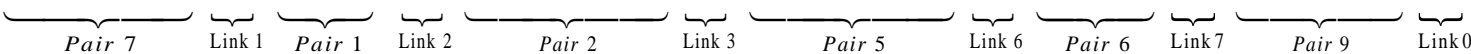
in matrix form:

Let us write symbolic formula of circuits' closure

$$
M_{1,1} \cdot M_{1,2} \cdot \ldots \cdot M_{1, n}=E, \quad l=1,2,
$$

where: $M_{1, a}=P_{1, a} \cdot G_{1, a}, \quad P_{1, a}$ is matrix of kinematic pair $(l, a), G_{1, a}$ is matrix of binary $\operatorname{link}(l, a)$. The symbol $[E]$ (unity matrix) indicates that the circuit is closed.

In order to analyze the movements of the parallel manipulator an iterative process is used, whereby the unknown dependent variables $r_{l, a}$ are expressed in terms of their initial estimates $\bar{r}_{l, a}$ and disparities $d r_{l, a}$ by the formula:

$$
r_{l, a}=\bar{r}_{l, a}+d r_{l, a}, \quad\left(r_{l, a}=1,2, \ldots, n-m\right)
$$

Let us represent each matrix $M_{1, a}$ in formula (3) in the form of a Taylor series expansion, limited to the first two of its components:

$$
M_{l, a} \approx \bar{M}_{l, a}+\sum_{k=1}^{3} \frac{\partial M_{l, a}}{\partial r_{l, a}^{(k)}} d r_{l, a}^{(k)} .
$$

Let us introduce the matrix operator for various kinds of kinematic pairs $Q_{l, a}^{(k)}$, differentiating matrix:

$$
\frac{\partial M_{l, a}}{\partial r_{l, a}^{(k)}}=Q_{l, a}^{(k)} \cdot \bar{M}_{l, a},
$$

then,

$$
\begin{aligned}
& M_{l, a} \approx \bar{M}_{l, a}+\sum_{k=1}^{3} Q_{l, a}^{(k)} \cdot \bar{M}_{l, a} d r_{l, a}^{(k)} \approx \\
& \approx\left(E+\sum_{k=1}^{3} Q_{l, a}^{(k)} d r_{l, a}^{(k)}\right) \bar{M}_{l, a} .
\end{aligned}
$$

Considering the properties of the iterative process, all higher-order components $d r_{l, a}^{(k)} \cdot d r_{l, a}^{(k)}$ can be ignored. Taking the notation:

$$
\begin{gathered}
\sum_{k=1}^{3} B_{l, a}^{(k)}=M_{1,1} \cdot M_{1,2} \cdot \ldots \cdot M_{l, a-1} \cdot \sum_{k=1}^{3} Q_{l, a}^{(k)} \cdot M_{l, a} \cdot \ldots \cdot M_{1, n_{1}}, \\
D_{l, a}=\bar{M}_{1,1} \cdot \bar{M}_{1,2} \cdot \ldots \cdot \hat{M}_{l, a} \cdot \ldots \cdot \bar{M}_{1, n_{1}}
\end{gathered}
$$


$C_{l, a}=E-D_{l, a}$.

We will get matrix equation:

$$
\sum_{k=1}^{3} B_{l, a}^{(k)} \cdot d r_{(l, a)}^{(k)}=C_{l, a} ., \quad l=1,2 .
$$

The solution of the set of Eq. (11) satisfying the best root mean square criterion is:

$$
d r(l, a)=\left[B_{l, a}^{T} \cdot B_{l, a}\right]^{-1} \cdot B_{l, a}^{T} \cdot C_{l, a} .
$$

Having added the obtained values of deviations to their initial values, we will get more precise values of the time-varying parameters.

The position of the mobile platform relatively to $O X Y Z$ system of absolute coordinates can be defined by matrices $S_{C}, S_{D}, S_{G}$ :

$$
\begin{aligned}
& S_{C}=G_{07} \cdot M_{l, 1} \cdot M_{l, 2} \cdot M_{l, 3} \\
& S_{D}=G_{07} \cdot M_{l, 1} \cdot M_{l, 2} \cdot M_{l, 3}, \\
& S_{G}=G_{07} \cdot M_{l, 1} \cdot M_{l, 2} \cdot M_{l, 3}
\end{aligned}
$$

where: $\mathrm{G}_{07}$ is the transfer matrix between the $X_{7} Y_{7} Z_{7}$ coordinate system and the $O X Y Z$ absolute coordinate system.

\section{Inverse kinematic problem}

If the number of unknowns is equal to the number of equations, it is usually possible to find defined values of generalized coordinates. If the number of unknowns exceeds the number of equations, then different sets of generalized coordinates can match to the same position of the gripper. Finally, if the number of unknowns is less than the number of equations, the solution does not exist. Here, solving the inverse kinematic problem of tripod-type parallel manipulator we will always consider that the number of unknowns is equal to the manipulator's number of degrees of freedom.

In this work, alternate change of the generalized coordinates is performed for kinematic analysis, in order to minimize the norm between the position of the working body after changes in fixed internal coordinate and its target location.

In other words, using homogeneous transfer matrix of Wicker-Denavit-Hartenberg for spatial mechanism, the algorithm is being given to analyze the locations of the spatial arrangements, and a program of the computational solution to equations is reviewed. The developed method suiting to the algorithm has reliability and convergence, and in all cases, it permits to find a solution. It remains functionally operative even in special configuration of mechanism. Without additional changes, this algorithm allows us to find a unique solution. The algorithm is computationally efficient.

According to the inverse kinematic problem of present tripod-type parallel manipulator, among possible relative motions sought quantities are constant motion in all rectilinear and cylindrical kinematic pairs.

\section{Discussion}

Currently, there are some generic algorithms [1215], allowing the user to get an accurate solution of the inverse kinematic problem for a general mechanism. However, solving of the inverse problem in real-time is now quite difficult for general manipulation robot.

The inverse kinematic problem not always can be solved analytically. The estimation of efficiency of various numerical iterative methods for the analysis of the locations of the parallel manipulator is currently produced in general form. These methods can be classified as five different approaches:

1. Solution to kinematic equation by the NewtonRaphson method.

2. The speed in the kinematic pairs is calculated of the linear relation of the actuating device rate of change and speed of the kinematic pairs. problem

3. Numerical solution of the inverse kinematic

4. Alternate change of the generalized coordinates, in order to minimize the norm between the position of the working body after changes in fixed internal coordinate and its target location.

5. Application of the continuation method for solving sets of algebraic equations.

If first-order integration scheme is used, the second approach leads to the above-mentioned Newton-Raphson method. In general, both these methods require precise initial values. It should be noted that concerning to the manipulators in configurations that are close to specific position; these algorithms are characterized by low reliability and low stability and efficiency. Modified algorithm of the Newton-Raphson method and the integral method of forward corrective actions that increase their reliability and stability, allow to find a solution, provided by the feasibility of this configuration. They are also applicable to configurations that are close to specific position, by using some predetermined convergence criterion. While the modified method of forward corrective actions (MFCA) requires very accurate initial values of generalized coordinates, a modified Newton-Raphson method (MNR) can be used for the initial values of generalized coordinates of arbitrary precision. However, both of the MFCA and the MNR algorithms are inefficient when the manipulator gets to a special configuration. It was shown that the MFCA is computationally several times faster than MNR. The value of working body velocities is also should be known. The main modification of the basic algorithm for both numerical methods (MNR and MFCA) concerns the criterion selection of descending grade in the iterative process.

The basic algorithm in the fifth approach is guaranteed to determine sets of all possible solutions for the generalized coordinates. However, it should be noted that if the manipulator is in some configuration, it should pass through a certain special position in order to change this configuration.

\subsection{Numerical solutions of the direct kinematic problem}

To implement six Wicker-Denavit-Hartenberg parameters, the tripod-type parallel manipulator with the 
corresponding constant geometry and kinematic features $a_{l, a}, \alpha_{l, a}, b_{l, a}, \beta_{l, a}, c_{l, a}, \gamma_{l, a}$ can be used (Fig. 1).

Present parallel manipulator has $n=9$ kinematic pairs, $n_{l}=7$ links, $m=6$ generalized coordinates, and $L=2$ circuits. The first circuit is formed by links that are limited by kinematic pairs 1-2-3-6-5-4, and the second circuit by kinematic pairs 1-2-3-9-8-7. ICS OXYZ is rigidly connected to the zero state, the beginning of which is in the 7-th kinematic point.

To evaluate the efficiency of the developed algorithm, a computer program for the solution of the direct kinematic problem of the tripod-type parallel manipulator was developed.

The values of the unknown parameters would be defined by the iterative method. It is considered that the removal gripper has a set length. We divide time into equal narrow intervals. Received generalized coordinates of required accuracy for one point in time, we can use them as an initial approximation to the next position, etc., until achieve the end of overall time interval.

The result of numeric computation includes the change of points in links of tripod-type parallel manipulator with rectilinear, cylindrical, and spherical kinematic pair. Graphs of kinematic characteristics change depending on operation time presented in Fig. 2.

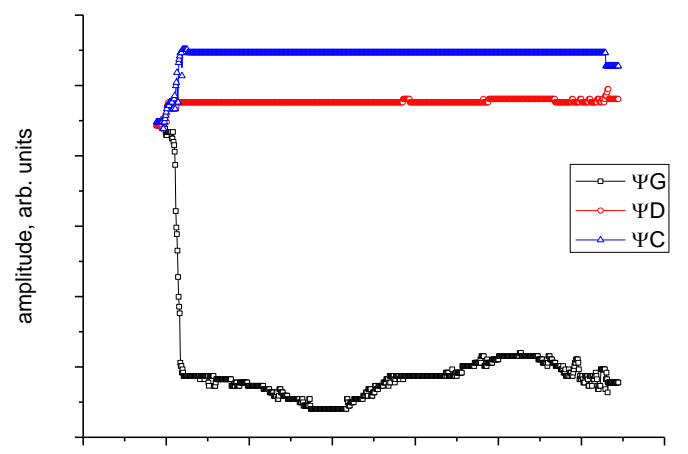

a

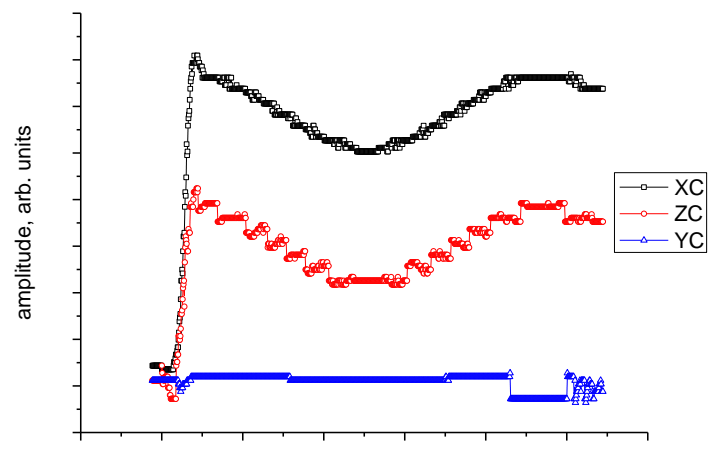

b

Fig. 2 Changes in coordinates of points and angles of rotation of the tripod-type parallel manipulator mobile platform. The horizontal axis represents time in seconds, the vertical axis - the coordinates of points and angles of rotation of the mobile platform.

\subsection{Numerical solutions of the inverse kinematic problem}

In order to check the efficiency of the WickerDenavit-Hartenberg iterative method a computer program for multicircuit tripod-type parallel manipulator with high degrees of freedom with the relevant geometric and kinematic features (Fig. 1) was developed due to sequential algorithm. There are two variants for the numerical calculation:

\subsubsection{First variant}

Based on the technical requirement, kinematic scheme and geometric parameters of the parallel manipulator were selected, and they take the following values:

$$
\begin{aligned}
& l_{1}=l_{3}=l_{5}=l_{4}=l_{6}=l_{2}=0.8 ; l_{7}=0.08 ; \\
& s_{2}=s_{5}=s_{8}=\sqrt{\left(4 l_{1}^{2}+l_{1}^{2} / 4\right)}-2 l_{1} ; \\
& s_{1}=s_{4}=s_{7}=l_{1} / 2 ; c_{1,10,11}=c_{2,10,11}=l_{1} ; \\
& c_{1,12,1}=l_{7} / 2 ; \gamma_{1,6,7}=300^{\circ} ; \beta_{1,9,10}=60^{\circ} ; \\
& a_{1,6,7}=a_{2,6,7}=a_{2,12,1}=l_{7} ; \\
& b_{1,2,3}=b_{1,3,4}=b_{1,9,10}=b_{2,2,3}=b_{2,3,4}=b_{2,9,10}=l_{1} ; \\
& b_{1,12,1}=l_{7} \sqrt{3} / 2 .
\end{aligned}
$$

Here: $S_{2}, S_{5}, S_{8}$ is the displacement of pairs 2, 5, 8; $S_{1}, S_{4}, S_{7}$ is the displacement of pairs $1,4,7$.

In the process of simultaneous movement of the drive components $1,7,2,5,8$, and alternate movement of drive component 4 , in direct kinematic problem for a given generalized coordinates, the angles of rotation and the coordinates of the points of links of parallel manipulator, were founded.

According to the solution of inverse kinematic problem, generalized coordinates were found They define the requirements for the drive components and control system for the selection and design of drives and the entire mechanism.

Using the found specified values of generalized coordinates, the problem of the manipulator's movement (direct problem) is being solved, i.e. kinematic synthesis is being held. We make sure that the technical requirements are fulfilled or we make the decision to change the structure of the manipulator or control system.

\subsubsection{Second variant}

Based on the technical requirement, kinematic scheme and geometric parameters of the parallel manipulator were selected, and they take the following values:

$$
\begin{aligned}
& l_{1}=l_{3}=l_{5}=l_{4}=l_{6}=l_{2}=0.6 ; l_{7}=0.06 ; \\
& s_{2}=s_{5}=s_{8}=\sqrt{\left(4 l_{1}^{2}+l_{1}^{2} / 4\right)}-2 l_{1} ; \\
& s_{1}=s_{4}=s_{7}=l_{1} ; \\
& c_{1,10,11}=c_{2,1-, 11}=l_{1} ; c_{1,12,1}=l_{7} / 2 ; \gamma_{1,6,7}=300^{\circ} ; \\
& a_{1,6,7}=a_{2,6,7}=a_{2,12,1}=l_{7} ; \\
& b_{1,2,3}=b_{1,3,4}=b_{1,9,10}=b_{2,2,3}=b_{2,3,4}=b_{2,9,10}=l_{1} ; \\
& b_{1,12,1}=l_{7} \sqrt{3} / 2 .
\end{aligned}
$$

Here: $S_{2}, S_{5}, S_{8}$ is the displacement of pairs 2, 5, 8; $S_{1}, S_{4}, S_{7}$ is the displacement of pairs $1,4,7$. 
In the process of simultaneous movement of the drive components $1,7,2,5,8$, and alternate movement of drive component 4 , in direct kinematic problem for a given generalized coordinates, the angles of rotation and the coordinates of the points of links of parallel manipulator, were founded.

According to the solution of inverse kinematic problem, generalized coordinates were founded. They define the requirements for the drive components and control system for the selection and design of drives and the entire mechanism.

Using found specified values of generalized coordinates, the problem of the manipulator's movement (direct problem) is being solved, i.e. kinematic synthesis is being held, see Fig 3 . We make sure that the technical requirements are fulfilled or we make the decision to change the structure of the manipulator or control system.

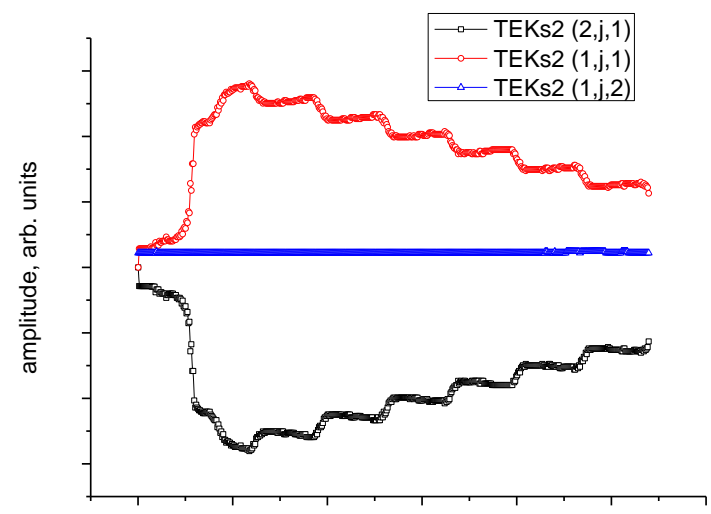

a

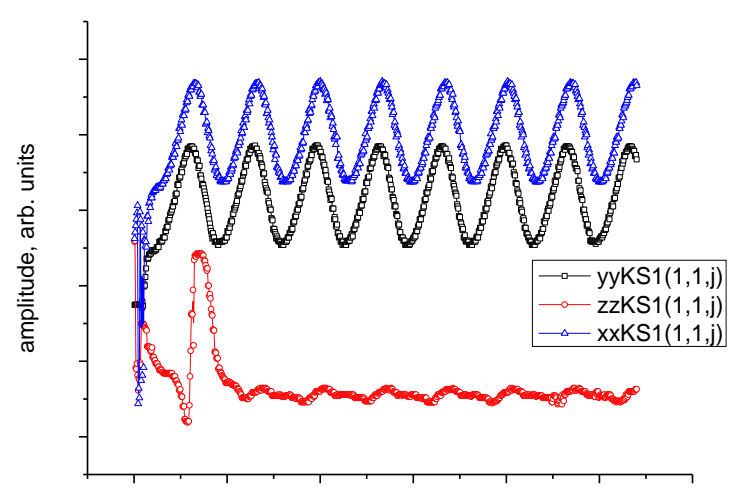

b

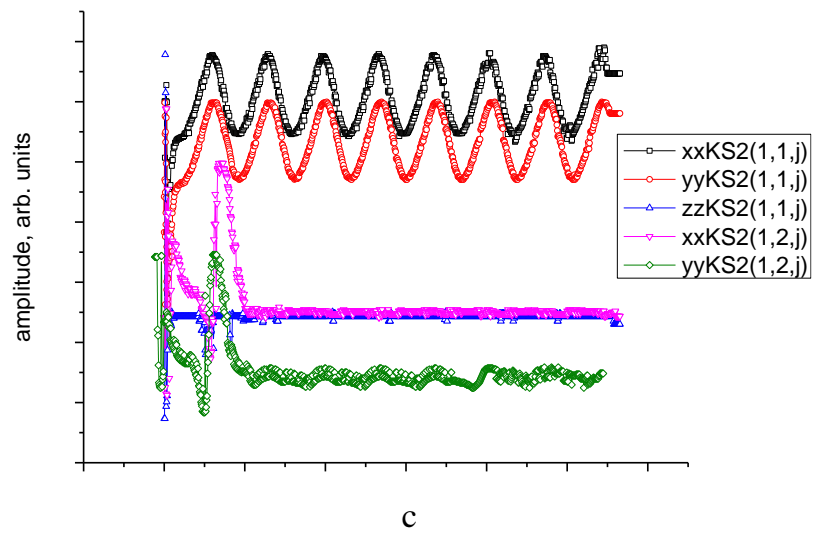

Fig. 3 Location changes (a), of the rotation angles (b) and the coordinates of articulation points (c) in links of the parallel manipulator over time (the kinematic synthesis)
Positional accuracy of industrial robots is one of the most important problems in robotic engineering. The robot accuracy is determined positioning inaccuracy of characteristic gripper point and by inaccuracy in angular orientation of the gripper. The positioning inaccuracy is determined by technological offset of manipulator links size, splits in kinematic pairs and drive components, deformations of links (elastic and temperature), as well as by errors of operating system and feedback probes.

The solution of the direct kinematic problem allows us to calculate the error in process position of the mobile platform centre. To solve the direct kinematic problem geometric parameters of the manipulator, have to be known (such as the radius of the base of the mobile platform, the maximum and minimum out-in of manipulator arms, the height of manipulation robot), and for the solution of the inverse kinematic problem, it is enough to know the mechanical trajectory of the platform.

\section{Conclusion}

In this work, the design of tripod-type parallel manipulator consisting of executive and close kinematic chains is proposed. This article utilises methods for solving direct and inverse kinematic problems of the tripod-type parallel manipulator based on generalized parameters Sheth-Wicker.

This article applies an algorithm of application program for multi-optional computer calculations of direct and inverse kinematic problems of the tripod-type parallel manipulator. Analysis of the solution of direct kinematic problem for tripod-type parallel manipulator tripod have shown that the choice of the initial parameter values of the parallel manipulator affects the distortion or accuracy of output parameter.

Analysis of the solution of inverse kinematic problem for tripod-type parallel manipulator tripod have shown that there is the need to avoid such position of the manipulator whereby the initial approximation is close to the desired solution.

The developed algorithms and computer-generated programs for synthesizing and analysing the tripod-type parallel manipulator are adaptable to spatial mechanism design. These mechanisms are characterised by parallel kinematics with high degrees of freedom, an arbitrary number of edges, and an arbitrary arrangement of segments with different kinematic pairs that can be used in many ways. For example, mathematics and software have been optimized for the task of stabilizing the camcorder of an unmanned aerial vehicle.

\section{References}

1. Quiter, M. C; Anderson, V. J. 2001. A proposed method for determining shrub utilization using low altitude/large scale aerial imagery, Journal of Range Management 54: 378-381.

2. Rango, L. A.; Laliberte, A.; Herrick, J. E.; Winters, C.; Havstad, K.; Steele, C.; Browning, D. 2009. Unmanned aerial vehicle-based remote sensing for rangeland assessments, monitoring and management, Journal of Applied Remote Sensing 3: 1-15.

3. Breckenridge, R. P.; Dakins, M. E. 2011. Evaluation of bare ground on rangelands using unmanned aerial vehicles: a case study, GIScience \& Remote Sensing 48: 74-85. 
4. Hardin, P. J.; Jackson, M. W. 2005. An unmanned aerial vehicle for rangeland photography, Rangeland Ecology \& Management 58: 439-442.

5. Hardin, P. J.; Jackson, M. W.; Anderson, V. J.; Johnson, R. 2007. Detecting squarrose knapweed (Centaurea virgata Lam. ssp. Squarrosa Gugl.) using a remotely piloted vehicle: a Utah case study, GIScience and Remote Sensing 44: 203-219.

6. Rango L. A., Laliberte A, Steele C., Herrick J. E., Bestelmeyer B., Schmugge T., Roanhourse A., Jenkins V. 2006. Using unmanned aerial vehicles for rangelands: current applications and future potential, Environmental Practice 8: $159-168$.

7. Al'van, H. M.; Sloushh, A. V. 2003. The control of spatial motion platform with multiple degrees of freedom, Theory of mechanisms and machines 1: 63-69.

8. Volkomorov, S. V.; Karpenko, A. P.; Leletko, A. M. 2010. Optimization of linear and angular dimensions of single and two-section manipulators based on parallel kinematics, Science and Education: Electronic scientific and technical publication 08: 4-4.

9. Gwinnett, J. E. 1931. Amusement device. US Patent No. 1: 789,680

10. Gough, V. E. 1956-1957. Contribution to discussion of papers on research in Automobile Stability. Control and Tyre performance, Proc. Auto Div. Inst. Mech. Eng.: 392-394.

11. Stewart, D. 1965. A Platform with Six Degrees of Freedom, UK Institution of Mechanical Engineers Proceedings 180(15): 1965-1966.

12. Morell, A.; Tarokh, M.; Acosta, L. 2013. Solving the forward kinematics problem in parallel robots using Support Vector Regression, Engineering Applications of Artificial Intelligence 26(7): 1698-1706.

13. Plitea, N.; Szilaghyi, A.; Pisla, D. 2015. Kinematic analysis of a new 5-DOF modular parallel robot for brachytherapy, Robotics and Computer-Integrated Manufacturing 31: 70-80.

14. Trang, T. Th.; Li, W. G.; Pham, Th. L. 2015. A new method to solve the kinematic problem of parallel robots using an equivalent structure, Applied Mechanics \& Materials 789: 643-651.
15. Wang, M.; Ceccarelli, M. 2015. Topology search of 3-DOF translational parallel manipulators with three identical limbs for leg mechanisms, Chinese Journal of Mechanical Engineering 28(4): 666-675.

\section{B. Nurakhmetov, K. Sartayev, Zh. Myrzageldiyeva \\ DIRECT AND INVERSE KINEMATIC PROBLEMS OF TRIPOD-TYPE PARALLEL MANIPULATOR}

S u m m a r y

The purpose of the work is to study the structural features of the tripod-type parallel manipulator as well as to solve direct and inverse kinematic problems for such a manipulator. In order to determine the fixed position of the movable platform at the generalized coordinates in the direct kinematic problem and at the unknown generalized coordinates in the inverse kinematic problem, multi-sweep kinematic procedure of multistage core parallel manipulator with different kinematic pairs was applied. The developed algorithms and computer-generated programs for synthesizing and analysing the tripod-type parallel manipulator are adaptable to spatial mechanism design. These mechanisms are characterised by parallel kinematics with high degrees of freedom, an arbitrary number of edges, and an arbitrary arrangement of segments with different kinematic pairs that can be used in many ways. For example, the foregoing can be used in machine processing of the inner surfaces of the cavities of complex shape, during maintenance and reclamation work in pipeline, during surgical operations in medicine etc.

Keywords: robotic engineering, direct and inverse kinematic problem, multi linkage design.

Received November 20, 2017 Accepted October 14, 2019 